\title{
Letter in Response to "HIF-Prolyl Hydroxylase Inhibitors: Confirmed Efficacy with Uncertain Safety"
}

\author{
Hiroyasu Yamamoto $^{a}$ Kiyoshi Nobori $^{\mathrm{b}}$ Yoshimi Matsuda $^{\mathrm{b}}$ Yasuhiro Hayashi ${ }^{\mathrm{c}}$ \\ Takanori Hayasaki ${ }^{c}$ Tadao Akizawa $^{d}$ \\ aDivision of Nephrology and Hypertension, Department of Internal Medicine, The Jikei University School of \\ Medicine, Tokyo, Japan; besearch and Development Japan, Bayer Yakuhin, Ltd., Osaka, Japan; \\ 'Medical Affairs and Pharmacovigilance, Bayer Yakuhin, Ltd., Osaka, Japan; dDivision of Nephrology, \\ Department of Medicine, Showa University School of Medicine, Tokyo, Japan
}

Dear Editor,

We thank Roger and Coyne [1] for their insight in our articles presenting efficacy and safety results of molidustat for the treatment of renal anemia in the non-dialysis (ND) correction and maintenance MIYABI phase 3 active-controlled trials. As discussed in our 2 articles, we agree with Roger and Coyne that, based on current results, longer studies with a larger population - including post-authorization surveillance studies such as the Longterm Special Drug Use Investigation of Molidustat for Patients With Renal Anemia study (NCT04899661) - are needed to understand the association between hypoxiainducible factor prolyl hydroxylase inhibitors (HIF-PHIs) and major adverse cardiovascular events (MACEs) as the next step toward the safe use of this promising drug class.

Based on the phase 3 data available, there is no robust evidence suggesting that the safety profile of molidustat is inferior to that of darbepoetin. Indeed, MACEs identified in patients treated with molidustat in the 2 trials featured here have been considered unrelated to the study drug by the investigators. The overall imbalance in MACEs between molidustat and darbepoetin in the combined analysis of the ND correction and maintenance trials presented by Roger and Coyne needs to be interpreted in the context of several limitations, including the low number of MACEs and the short duration of the trials. In addition, we believe that the imbalance in baseline characteristics reported in the ND correction study between molidustat and darbepoetin contributed to the higher incidence of MACEs observed in the combined analyses [2]. Accordingly, the Japanese Pharmaceutical and Medical Devices Agency highlighted the need for further investigations before drawing definitive conclusions regarding the risk of MACEs related to molidustat.

As mentioned by Roger and Coyne [1], a trend toward increased MACEs compared with darbepoetin has also been observed in ND studies for the HIF-PHI vadadustat, approved in Japan. However, the risk of MACEs associated with roxadustat was similar to that of darbepoetin in the 2-year DOLOMITES study in patients not receiving dialysis. Thus, it remains to be determined whether the potential safety concerns are class related or drug specific. Those potential concerns may be partly related to the different hemoglobin targets used in the ND and hemodi- 
alysis (HD) trials. In the molidustat MIYABI program, MACEs were observed in 5.5\% of patients in the ND studies (hemoglobin target: $11-13 \mathrm{~g} / \mathrm{dL}$ ) versus $2.8 \%$ of those in the HD trials (hemoglobin target: $10-12 \mathrm{~g} / \mathrm{dL}$ ) [3]. We agree that results from these 2 trials warrant the need for further monitoring of MACEs in patients treated with molidustat, and special attention should be paid to the use of HIF-PHIs in patients with cardiovascular diseases or history of thromboembolic events, as recommended by the Asian Pacific Society of Nephrology [4]. Nevertheless, these are still early days to draw definitive conclusions about the safety profile of HIF-PHIs.

\section{References}

1 Roger SD, Coyne DW. HIF-Prolyl Hydroxylase Inhibitors: Confirmed Efficacy with Uncertain Safety. Am J Nephrol. 2021.

2 Yamamoto H, Nobori K, Matsuda Y, Hayashi Y, Hayasaki T, Akizawa T. Efficacy and safety of molidustat for anemia in ESA naive nondialysis patients: a randomized, phase 3 trial. Am J Nephrol. 2021;1-13. Epub ahead of print.
3 Akizawa T, Takashi Y, Nobori K, Matsuda Y, Hayashi Y, Hayasaki T, et al. Molidustat for Japanese patients with renal anemia receiving dialysis. Kidney Int Rep. 2021;6(10):2604-616.

4 Yap DYH, McMahon LP, Hao CM, Hu N, Okada H, Suzuki Y, et al. Recommendations by the Asian Pacific society of nephrology (APSN) on the appropriate use of HIF-PH inhibitors. Nephrology. 2021 Feb;26(2):105-18. 